\title{
The Cost of Patients with Chronic Kidney Failure Before Dialysis: Results from the IRIDE Observational Study
}

\author{
Claudio Jommi $^{1,2} \cdot$ Patrizio Armeni $^{2} \cdot$ Margherita Battista $^{1} \cdot$ Paolo di Procolo $^{1}$. \\ Giuseppe Conte $^{3}$ - Claudio Ronco ${ }^{4}$ - Mario Cozzolino ${ }^{5}$ Anna Maria Costanzo ${ }^{6}$. \\ Umberto di Luzio Paparatti ${ }^{6}$. Gabriella Concas ${ }^{7} \cdot$ Giuseppe Remuzzi $^{8}$. \\ On behalf of the IRIDE Study Group
}

Published online: 4 December 2017

(C) The Author(s) 2017. This article is an open access publication

\begin{abstract}
Background Chronic kidney disease (CKD) is an important public health problem. Most of the evidence on its costs relates to patients receiving dialysis or kidney transplants, which shows that, in these phases, CKD poses a high burden to payers. Less evidence is available on the costs of the predialytic phase.

Objective The aim of this study was to estimate the annual cost of patients with CKD not receiving dialysis treatment, using the Italian healthcare system perspective and a prospective approach.
\end{abstract}

Claudio Jommi

claudio.jommi@uniupo.it

1 Department of Pharmaceutical Sciences, Università del Piemonte Orientale, Largo Donegani 2, Novara, Italy

2 Centre for Research in Health and Social Care Management (CERGAS), SDA School of Management Bocconi, Via Roentgen 1, Milan, Italy

3 Nephrology Division, School of Medicine, Second University of Naples, Via Santa Maria di Costantinopoli, 104, Naples, Italy

4 Department of Nephrology, Dialysis and Transplantation, International Renal Research Institute of Vicenza (IRRIV), San Bortolo Hospital, Viale Ferdinando Rodolfi, 37, Vicenza, Italy

5 Renal Division, Department of Health Sciences, ASST Santi Paolo e Carlo, University of Milan, Via A. Di Rudinì, 8, Milan, Italy

6 Abbvie Srl, Campoverde di Aprilia, Via Pontina, 52, Aprilia, Latina, Italy

7 Struttura Complessa Territoriale Nefrologia e Dialisi-ASL 8 Cagliari, Via Is Mirrionis, 92, Cagliari, Italy

8 IRCCS, Istituto di Ricerche Farmacologiche Mario Negri, Via GB Camozzi 3, Ranica, Bergamo, Italy
Methods A 3-year observational study (December 2010September 2014) was carried out to collect data on resource consumption for 864 patients with CKD. Costs were estimated for both patients who completed the followup and dropouts.

Results The mean annual total (healthcare) cost per patient equalled €2723 (95\% confidence interval 2463.0-2983.3). Disease severity (higher CKD stage), multiple comorbidities, dropout status and belonging to the southern region are predictive of higher costs. Pharmaceuticals, hospitalisation, and outpatient services account for 71.5, 18.8 and $9.7 \%$ of total healthcare expenditure, respectively. Recent estimates of Italian costs of patients receiving dialysis are nine times the unit costs of CKD for patients estimated in this study. Unit costs at stage 5 CKD (the highest level of severity) equals 4.7 times the costs for patients at stage 1 CKD. Conclusion Despite its limitations, this study provides further evidence on the opportunity to invest in the first phases of CKD to avoid progression and an increase in healthcare costs. 


\section{Key Points for Decision Makers}

This 3-year observational study shows that healthcare costs of patients with chronic kidney disease (CKD) not receiving dialysis are, on average, $€ 2.7 \mathrm{k}$.

The last stages of CKD account for 17-22\% of unit healthcare costs of patients receiving dialysis. Policymakers should enhance all actions to postpone patients starting on dialysis; this would imply important savings for the healthcare system

The greater the severity of CKD, the higher the costs of patients with CKD. The more patients are kept at the initial stages, the higher the savings will be for the healthcare system.

Early management of patients with CKD in the predialysis stages, as well as the use of proper preventive treatments, may in fact slow the progression of kidney damage, including access to dialysis, with consequent savings of economic resources

\section{Background}

Chronic kidney disease (CKD) is an important public health problem. In the US, CKD is the ninth leading cause of death and affects more than $10 \%$ of the adult population. Total US Medicare expenditure for CKD (excluding drugs) accounts for $6 \%$ of all Medicare costs [1].

The literature relating to costs of CKD include different contributions for end-stage renal disease (ESRD) that requires renal replacement therapy (RRT) in the form of dialysis or transplantation. These costs represent $2-3 \%$ of the annual healthcare budget of public health systems [2]. The annual growth rate of dialysis costs has ranged from 6 through $12 \%$ over the past 2 decades in most developed countries [3]. In Italy in 2001, it was estimated that $1.8 \%$ of the total healthcare budget was spent on ESRD patients, representing $0.083 \%$ of the general population [4]. According to more recent estimates, total healthcare spending on ESRD has reached $€ 2.1$ billion in 2010 (i.e. $1.9 \%$ of the healthcare budget) and healthcare costs of patients receiving dialysis range from $€ 29.8 \mathrm{~K}$ to $€ 43.8 \mathrm{~K}$ depending on the techniques used (peritoneal dialysis vs. haemodialysis) [5].

Costs in the earlier stage of CKD before dialysis are less investigated. A paper published in 2009 on US Medicare costs shows that the average cost per patient receiving dialysis is 2.5 times the costs of patients with CKD, and the average healthcare costs of patients with CKD is three times the unit cost of patients covered by Medicare [6]. More recent research on the cost of CKD in Australia, relying on a national longitudinal population-based study of Australian adults with diabetes aged $\geq 25$ years, shows a significant difference in the per-person annual direct healthcare costs by CKD status-from $\$ 1829$ for those without CKD to $\$ 14,545$ for those with stage 4 or $5 \mathrm{CKD}$, where stages refer to decreasing glomerular filtration rate (GFR) and range from 1 to 5 [7].

Another retrospective study on the population with type 2 diabetes was carried out in Germany. The costs for patients in stages 4 and 5 were 1.97 and 3.5 times, respectively, the costs of patients in stage 3 [8]. In Italy, the evidence on predialysis costs is also limited. A recent study carried out in Tuscany ( $6.2 \%$ of the Italian population) in 2012 and 2013, and focusing on stage 4 and 5 CKD, produced estimated direct medical costs of $€ 4352$, i.e. $44 \%$ of the overall social cost (€9855) [9]. A retrospective analysis on prescriptions by 124 general practitioners (GPs) for 12,400 patients has estimated 2010 mean healthcare costs per patient ranging from $€ 450$ in stages $1-2$, to $€ 3292$ in stage 5 [5]. More recent retrospective studies have been carried out on regional databases and have investigated the unit cost per patient in the predialysis and dialysis phases. The first study, in the Piedmont region $(7.2 \%$ of the Italian population), compared incident-to-dialysis patients observed for the 12 months before dialysis entrance $(€ 11,123)$ and established dialysis patients $(€ 53,764)$ [10]. The second analysis was carried out in the Lombardy Region (16.5\% of the Italian population) and found a unit healthcare cost per patient of $€ 5239, € 12,303$ and $€ 38,821$ in the 12- to 24-month period before dialysis, 0-12-month period before dialysis, and the first year of dialysis, respectively [11].

However, none of these studies investigated the healthcare costs of patients with CKD, using a prospective approach and relying on data from specialist centres, which may provide a more complete collection of healthcare resources used than GPs.

\section{Research Question and Methods}

This research aims at filling the gap in the literature, i.e. estimating the annual costs of patients with CKD not receiving dialysis treatment, using the Italian healthcare system perspective and relying on a prospective multicentre approach. The costs of patients with CKD includes the costs of CKD and the costs of comorbidities that are 
possible consequences of $\mathrm{CKD}$, but not the costs of comorbidities not linked to the disease.

Patients aged $>18$ years with CKD (as defined based on the Kidney Disease Outcomes Quality Initiative (K-DOQI) guidelines [12]) in the predialysis phase were included in the study. Patients with comorbidities with a life expectancy of $<1$ year (e.g. advanced-phase malignancies, advanced liver disease), patients enrolled in a clinical trial (interventional study) receiving erythropoietin, vitamin D or phosphate binders at the start of the survey, and renal transplant recipients were not included in the study. Dropouts included patients who had started dialysis or died during the follow-up period, who had withdrawn consent, and who had been lost to follow-up.

Data on resource consumption were collected through an electronic case record form (e-CRF). Patients were recruited in 24 centres (originally 26 , but in one centre the follow-up of all patients was interrupted for organisational issues, and one centre did not enrol any subjects) in 15 of the 21 Italian healthcare regions. Participating centres were selected according to the following criteria: (1) specialisation in nephrology; and (2) more than 20 patients visited the centre per week. Patients were consecutively recruited in order to limit selection bias. Sample size was determined on the grounds of alleged secondary hyperparathyroidism outcome in stage 4 patients since no other epidemiologic data had been reported in the literature at the time of the study design.

The follow-up lasted 3 years. For all participants, defined as those patients who met the inclusion/exclusion criteria after signing the informed consent, clinical and healthcare consumption data were recorded. The recruitment phase started on December 2010 and finished in September 2011. The follow-up phase was terminated in September 2014.

The study was also designed to collect data on productivity loss; however, despite 321 patients $(36.4 \%)$ being 25-65 years of age, working loss days were collected for nine patients only. Hence, productivity-loss data were not included in the analysis.

The physician responsible filled in the form every 6 months, up to 36 months. Responders were asked to collect data on (1) molecules, daily dosage, and treatment days; (2) outpatient services, including visits, diagnostic procedures and laboratory tests; and (3) inpatient services and the relevant Diagnosis-Related Group (DRG) code.

Drug unit costs were calculated as a 2014 mean unit price per dose (Drugs National Formulary), considering different products per molecule (the e-CRF did not allow responders to quote the brand name of the prescribed product) and different possible distribution systems. In fact, some drugs (e.g. new antidiabetic drugs or epoetins) may be distributed as follows.
- By community pharmacies (ordinary distribution): list price (net of discounts and co-payment) is paid by the Italian National Health Service (NHS).

- Directly by health authorities: ex-factory price, net of local discounts, is paid by the NHS. Local discounts were not available and gross ex-factory price was used.

- By community pharmacies on behalf of health authorities: ex-factory price, net of local discounts is paid by the NHS; pharmacies also receive a remuneration, as defined by local agreements. Since many responders did not distinguish between distribution by health authorities and distribution by community pharmacies on behalf of health authorities, we did not include the remuneration paid to pharmacies; local discounts were not available and gross ex-factory price was used.

For ordinarily distributed molecules with at least one generic available, we used the minimum price per dose since the NHS reimburses this cost.

Estimates of unit costs of ordinary and same-day (dayhospital) hospitalisations relied on the relevant 2012 national fee-for-service [13]. Extra fees per day of stay over the thresholds (maximum length of stay per DRG) were considered. Outpatient services were also monetized using the national fee-for-service [13].

If the patient's follow-up lasted over 3 years (the actual follow-up period could have been longer than 36 months if the time interval between two visits was longer than 6 months) or less than 3 years but more than 1 year (some patients dropped out), the annual cost per patient was estimated as 'daily cost $\times 365$ '. However, if the patient's follow-up lasted less than 1 year (patients dropped-out), the annual cost was estimated as "daily cost $x$ follow-up days'.

We performed descriptive statistics on unit costs per patient according to (1) patients' status, i.e. completed follow-up or dropout; (2) starting-level disease severity, measured through the GFR, ranging from 1 (lower CKD stage) to 5 (higher CKD stage); (3) sex and age of patients-10 age-group intervals were considered (18-24, 25-34, 35-44, 45-54, 55-64, 65-74, 70-74, 75-79, 80-84, over 84 years); (4) geographical areas (northern region, including Piedmont and Aosta Valley, Liguria, Lombardy, Veneto, Friuli Venezia Giulia; central region, including Tuscany, Marche, Latium, Abruzzo and Molise ${ }^{1}$; and southern region, including Campania, Apulia and Basilicata, and Sardinia; (5) presence of comorbidities (hypertension/diabetes/dyslipidaemia were the most frequent); and (6) proteinuria at the date of recruitment. Statistical

\footnotetext{
1 Abruzzo and Molise are usually considered southern regions but we preferred to include them in the central region group because they are more similar to that group.
} 
significance of differences between values was tested using the Kruskal-Wallis test.

The regression was performed using a linear model ordinary last squares (OLS) with robust standard error estimation to allow heteroskedasticity in residuals. The explanatory variables were geographical area (north, centre, south), age-group class, sex, set of comorbidities (also interacted with one another), severity at enrolment, presence of proteinuria at enrolment, and total time of enrolment.

\section{Results}

Healthcare cost analysis was performed on 864 patients, i.e. 884 patients recruited, net of 16 patients from one centre that had not completed follow-up and 4 patients whose costs were not filled in on the e-CRF. Overall, 586 patients completed the follow-up, whereas 278 patients interrupted the study before the last visit (32.2\%; ranging from $51.4 \%$ in Veneto and $18 \%$ in Apulia and Basilicata) (Table 1). Among the dropouts, $36.0 \%$ of patients began dialysis treatment, $34.5 \%$ were lost to follow-up and $25.2 \%$ died. The study population mainly consisted of males $(59.7 \%)$, and patients over 65 years of age accounted for $63.2 \%$ of the study population $(18.6 \%$ were over 80 years of age); mean age was 66.3 years ( \pm 14.6 years). At the time of enrolment, mean time from diagnosis was 7.0 years ( \pm 7.9 years) and only $0.7 \%$ of patients were at their first nephrologist visit (data not reported in Table 1). Patients with a starting GFR level in stages 1, 2, 3, 4 and 5 totalled $68,156,355$ (167 in stage $3 \mathrm{a}$ and 188 in stage $3 \mathrm{~b}$ ), 206, and 79 , respectively. Hypertension, hypercholesterolemia and diabetes were the most frequent comorbidities, with $89.1 \%$ of patients experiencing at least one of these comorbidities and 107 patients $(12.4 \%)$ being affected by all three.

Mean annual total (healthcare) cost per patient equalled $€ 2723$ (Fig. 1). All differences between values (status of patients, age, disease progression, comorbidities, starting GFR level, proteinuria at recruitment, and region) were found to be statistically significant according to the Kruskal-Wallis test, apart from the difference in unit costs between the male and female populations.

The unit costs of patients who dropped out were almost double those of patients who completed the follow-up. Dropped-out patients starting dialysis and dead patients during the follow-up period showed the highest mean annual cost, confirming that proximity to dialysis and death raises costs.

Disease progression produces an increase in costs. The higher the starting CKD stage, the higher the healthcare cost per patient. The largest difference between unit costs was found when comparing stage $3 \mathrm{~b}$ patients with stage $3 \mathrm{a}$ patients $(+56 \%)$ and stage 4 patients with stage $3 \mathrm{~b}$ patients $(+62 \%$ ) (Table 2). Stage 3 accounted for more than $40 \%$ of patients.

Proteinuria also seems an important explanatory variable of annual cost per patient. Costs for patients without a proteinuria at the recruiting date were $25 \%$ less than healthcare costs for patients with proteinuria (Table 2).

Patients with CKD often have other diseases, with diabetes, dyslipidaemia and hypertension being the most important and frequent. Hypertension seems to have a larger impact on unit costs; patients in whom renal failure is associated with hypertension alone and hypertension with diabetes show higher costs. However, not all comorbidities have an important effect on costs, e.g. unit costs of patients with diabetes are lower than the average unit cost (Table 2).

Mean annual cost per patient shows important variations across regions. On average, the cost per patient is higher in the southern and northern regions than in the central region (Table 2), despite the proportion of dropped out patients (who are, on average, costlier) being very similar across all areas.

Ageing produces a rise in costs (Fig. 2), however the growing trend shows an inflection point in the 70-74 age group. Costs are higher for males (€2779), but the difference in the female population is negligible $(+3 \%)$.

Pharmaceuticals, hospitalisation, and outpatients services account for $71.5,18.8$ and $9.7 \%$ of total healthcare expenditure, respectively (Table 3); hence, pharmaceuticals are the most important component of healthcare expenditure. Erythropoietins are the major component of drug expenditure (accounting for $36.4 \%$ of total drug expenditure), followed by antihypertensive drugs $(18.7 \%)$ and drugs for CKD [12.1\%, including vitamin D $(9.6 \%)$ and chelating agents (2.5\%)].

The contribution of hospitalisation to total costs is higher for patients who dropped out than for patients who completed the follow-up. More advanced is the starting CKD stage, the higher the proportion of inpatient costs (from $12.1 \%$ of total costs in stage 1 to $24 \%$ in stage 5 ) (Table 3).

Table 4 illustrates the results of the multiple regression model. The dependent variables are per capita total and annual drug expenditure. Explanatory variables are listed in column 1, while columns 2 and 3 include the reference variable and other variables, respectively. In columns 4 and 5, correlation coefficients for total healthcare and drug costs are reported. The explanatory power of the model is not particularly high ( $R$-squared ranges from 0.136 to 0.165). However, coefficients are significant for some variables; a higher starting CKD stage, together with the presence of three comorbidities, dropout status, and belonging to the southern region are significantly 
Table 1 Characteristics of the recruited patients

\begin{tabular}{|c|c|c|c|c|c|}
\hline Variable & $n($ total $=864)$ & $\%$ & Variable & $N($ total $=864)$ & $\%$ \\
\hline Follow-up completed vs. dropout & & & Age groups, years & & \\
\hline Follow-up completed & 586 & 67.8 & $18-24$ & 7 & 0.8 \\
\hline Dropout & 278 & 32.2 & $25-34$ & 25 & 2.9 \\
\hline Death & 70 & 8.1 & $35-44$ & 60 & 6.9 \\
\hline Dialysis & 100 & 11.6 & $45-54$ & 77 & 8.9 \\
\hline Lost to follow-up & 96 & 11.1 & $55-64$ & 149 & 17.2 \\
\hline Consent withdrawal & 7 & 0.8 & $65-69$ & 107 & 12.4 \\
\hline Inclusion criteria not satisfied & 3 & 0.3 & $70-74$ & 142 & 16.4 \\
\hline \multirow[t]{3}{*}{ Others } & 2 & 0.2 & $75-79$ & 145 & 16.8 \\
\hline & & & $80-84$ & 110 & 12.7 \\
\hline & & & $>84$ & 42 & 4.9 \\
\hline \multicolumn{6}{|l|}{ Region } \\
\hline Northern region & 384 & 44.4 & & & \\
\hline Piedmont and Aosta Valley & 85 & 9.8 & Starting CKD stage & & \\
\hline Liguria & 64 & 7.4 & 1 & 68 & 7.9 \\
\hline Lombardy & 165 & 19.1 & 2 & 156 & 18.1 \\
\hline Veneto & 35 & 4.1 & 3 & 355 & 41.1 \\
\hline Friuli VG & 35 & 4.1 & $3 a$ & 167 & 19.3 \\
\hline Central region & 279 & 32.3 & $3 b$ & 188 & 21.8 \\
\hline Tuscany & 98 & 11.3 & 4 & 206 & 23.8 \\
\hline Marche & 40 & 4.6 & 5 & 79 & 9.1 \\
\hline Latium & 101 & 11.7 & & & \\
\hline Abruzzo and Molise & 40 & 4.6 & Comorbidity & & \\
\hline Southern region & 201 & 23.3 & None & 94 & 10.9 \\
\hline Campania & 45 & 5.2 & Diabetes & 17 & 2.0 \\
\hline Apulia and Basilicata & 61 & 7.1 & Dyslipidaemia & 42 & 4.9 \\
\hline \multirow[t]{2}{*}{ Sardinia } & 95 & 11.0 & Hypertension & 275 & 31.8 \\
\hline & & & Diabetes + dyslipidaemia & 17 & 2.0 \\
\hline $\operatorname{Sex}$ & & & Diabetes + hypertension & 109 & 12.6 \\
\hline Female & 348 & 40.3 & Dyslipidaemia + hypertension & 203 & 23.5 \\
\hline \multirow[t]{5}{*}{ Male } & 516 & 59.7 & Diabetes + dyslipidaemia + hypertension & 107 & 12.4 \\
\hline & & & Starting proteinuria & & \\
\hline & & & Yes & 396 & 45.8 \\
\hline & & & No & 365 & 42.2 \\
\hline & & & Unkown & 103 & 11.9 \\
\hline
\end{tabular}

$C K D$ chronic kidney disease

correlated with both per capita healthcare and drug expenditure. These variables seem to explain more healthcare and drug expenditure than other variables (i.e. age, sex, and the presence of proteinuria at recruitment).

\section{Discussion}

The present study is the first analysis that has investigated the unit healthcare costs per patient with CKD before dialysis, using a national, observational, multicentre, prospective approach. The long follow-up period (3 years) and the involvement of healthcare centres in different regions allowed our analysis to produce a reasonable estimate of the actual unit cost in Italy, despite not stating that recruited patients represented all Italian patients with CKD before dialysis as no epidemiological data on CKD were available (incidence, prevalence, distribution of its various stages, etc.). However, the multicentric nature of the study and the fact that most regions were included, and recruited centres were selected on the grounds of a minimum number of patients who visited the centre, make this study more 
Fig. 1 Mean annual total cost per patient $(€)$. DO dropouts, $C V$ coefficient of variation. $* 12$ dropouts for reasons other than death, dialysis, and lost to follow-up

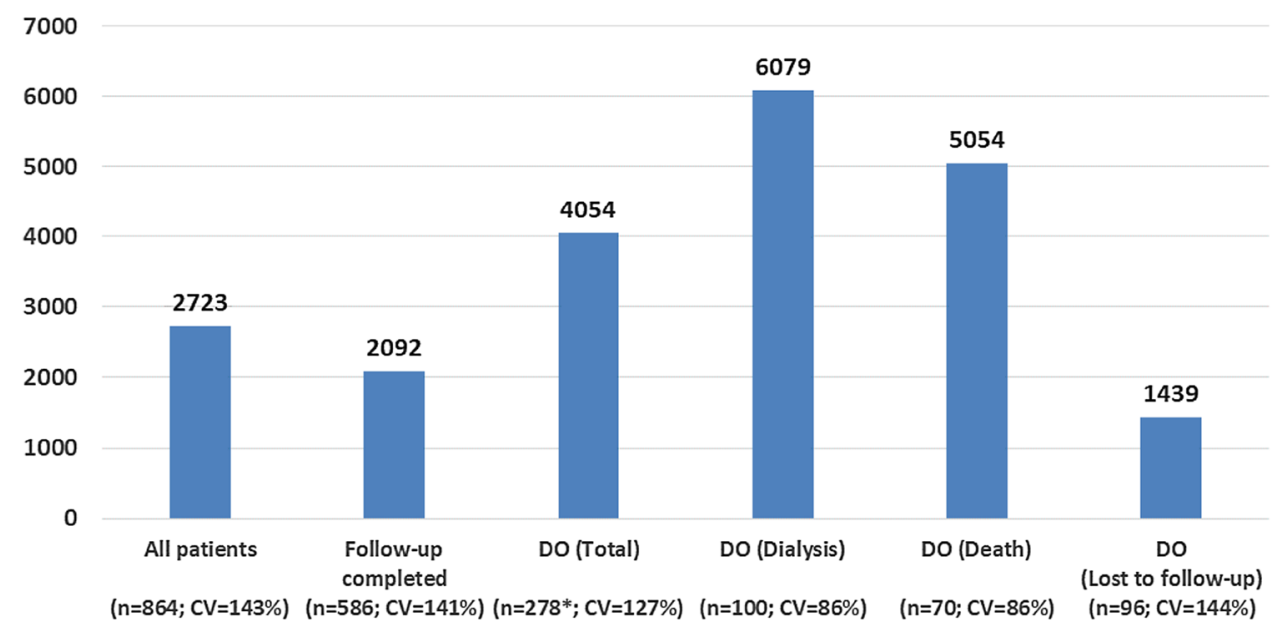

Table 2 Mean annual cost per patient according to their status (starting CKD level, proteinuria, comorbidities, geographic area) (€)

\begin{tabular}{|c|c|c|c|c|c|c|c|c|}
\hline & $N$ & Mean & Median & SD & $\begin{array}{l}\text { Coefficient } \\
\text { of variation }(\%)\end{array}$ & SE & $95 \% \mathrm{CI}$ & \\
\hline \multicolumn{9}{|l|}{ Starting $C K D$ stage } \\
\hline CKD 1 & 68 & 1169 & 865 & 1246 & 107 & 151.1 & 867.5 & 1470.8 \\
\hline CKD 2 & 156 & 1506 & 874 & 1845 & 123 & 147.7 & 1214.0 & 1797.7 \\
\hline CKD 3 & 355 & 2122 & 1074 & 2621 & 124 & 139.1 & 1848.5 & 2395.6 \\
\hline CKD $3 a$ & 167 & 1635 & 1019 & 1800 & 110 & 139.3 & 1359.9 & 1910.0 \\
\hline CKD 3b & 188 & 2555 & 1084 & 3119 & 122 & 227.5 & 2106.1 & 3003.5 \\
\hline CKD 4 & 206 & 4147 & 2325 & 5554 & 134 & 387.0 & 3384.1 & 4910.0 \\
\hline CKD 5 & 79 & 5453 & 3859 & 5293 & 97 & 595.5 & 4267.0 & 6638.2 \\
\hline \multicolumn{9}{|l|}{ Proteinuria at recruitment } \\
\hline Yes & 396 & 2937 & 1526 & 3781 & 129 & 190.0 & 2563.6 & 3310.7 \\
\hline No & 365 & 2100 & 1045 & 2865 & 136 & 150.0 & 1805.2 & 2395.1 \\
\hline Unknown & 103 & 4108 & 1840 & 6348 & 155 & 625.5 & 2867.4 & 5348.6 \\
\hline \multicolumn{9}{|l|}{ Comorbidities } \\
\hline Diabetes & 17 & 1472 & 840 & 1576 & 107 & 382.3 & 661.9 & 2282.7 \\
\hline Dyslipidaemia & 42 & 1845 & 1228 & 2003 & 109 & 309.0 & 1220.7 & 2468.8 \\
\hline Hypertension & 275 & 2675 & 1023 & 4197 & 157 & 253.1 & 2176.4 & 3172.8 \\
\hline Diabetes + dyslipidaemia & 17 & 2233 & 2014 & 1712 & 77 & 415.3 & 1353.0 & 3113.9 \\
\hline Diabetes + hypertension & 109 & 3083 & 1662 & 3230 & 105 & 309.4 & 2469.4 & 3695.9 \\
\hline Hypertension + dyslipidaemia & 203 & 2682 & 1406 & 3200 & 119 & 224.6 & 2239.5 & 3125.1 \\
\hline Diabetes + dyslipidaemia + hypertension & 107 & 3865 & 2038 & 4998 & 129 & 483.2 & 2906.9 & 4822.7 \\
\hline \multicolumn{9}{|l|}{ Regions } \\
\hline Northern & 384 & 2836 & 1237 & 4285 & 151 & 225.2 & 1964.5 & 2851.0 \\
\hline Central & 279 & 2408 & 1155 & 3761 & 156 & 218.7 & 2405.7 & 3265.7 \\
\hline Southern & 201 & 2946 & 1681 & 3236 & 110 & 228.3 & 2495.8 & 3396.0 \\
\hline
\end{tabular}

$C K D$ chronic kidney disease, $S D$ standard deviation, $S E$ standard error, $C I$ confidence interval

representative of the population than other studies focussed on a single region $[10,11]$.

Previous studies have investigated this topic using a retrospective approach applied to prescriptions by GPs, or single-region administrative databases. Despite the different approach, we found some commonalties with other studies, i.e. mean annual costs well below dialysis costs $[5,10,11]$ and an important increase in costs for more severe disease stages [5].

The study has some limitations. Unit drug costs were estimated as a weighted average price of all products sharing the same molecule and formulation (prescriptions 
Fig. 2 Mean annual cost per patient according to the different age groups (years) $(€)$

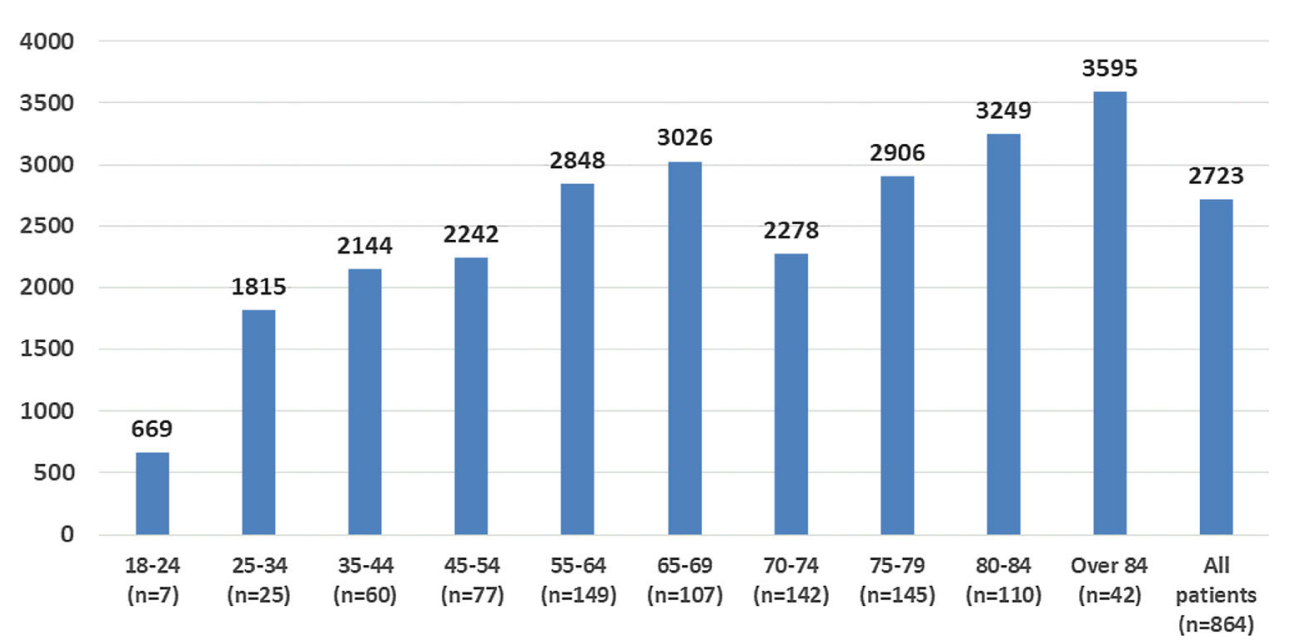

\begin{tabular}{lclrl}
\hline & Drugs $(\%)$ & Outpatient $(\%)$ & Inpatient $(\%)$ & Total $(\%)$ \\
\hline Patient status & & & & \\
All & $1946.8(71.5)$ & $263.8(9.7)$ & $512.5(18.8)$ & $2723.1(100.0)$ \\
Follow-up completed & $1590.1(76.0)$ & $259.6(12.4)$ & $241.8(11.6)$ & $2091.5(100.0)$ \\
Dropout & $2698.7(66.6)$ & $272.5(6.7)$ & $1083.2(26.7)$ & $4054.5(100.0)$ \\
Starting CKD stage & & & & \\
1 & $813.2(69.6)$ & $214.6(18.4)$ & $141.4(12.1)$ & $1169.2(100.0)$ \\
2 & $1096.7(72.8)$ & $225.7(15.0)$ & $183.4(12.2)$ & $1505.8(100.0)$ \\
3a & $1196.7(73.2)$ & $192.0(11.7)$ & $246.3(15.1)$ & $1634.9(100.0)$ \\
3b & $1790.1(70.1)$ & $227.6(8.9)$ & $537.0(21.0)$ & $2554.8(100.0)$ \\
4 & $3048.7(73.5)$ & $326.3(7.9)$ & $772.0(18.6)$ & $4147.0(100.0)$ \\
5 & $3686.4(67.6)$ & $456.3(8.4)$ & $1309.8(24.0)$ & $5452.6(100.0)$ \\
\hline CKD chronic kidney disease & & &
\end{tabular}

Table 3 Mean annual cost per patient for drugs, and outpatient and inpatient services $(€)$ differences in cost breakdowns. In our analysis, inpatient costs accounted for 18.6-24\% of total healthcare costs in the advanced stages (rising to more than $26 \%$ for patients who dropped out). Another study, which relied on a regional administrative database, has reported an incidence of hospitalisation costs higher than $75 \%$ in the 12 months preceding dialysis entrance for incident-to-dialysis patients [10].

\section{Conclusions}

Despite these limitations, this analysis has important policy implications. First, the annual healthcare costs per patient are approximately $10 \%$ of the healthcare costs of patients receiving dialysis [5]. If the social perspective was adopted, the difference could be even higher, considering working days lost and informal care for patients receiving dialysis. Hence, managing patients with CKD in order to avoid dialysis should be a priority to better allocate scarce resources. Second, one of the most important explanatory 
Table 4 Multiple regression analysis for total annual healthcare and drug costs per patient

\begin{tabular}{|c|c|c|c|c|}
\hline Variables & $\begin{array}{l}\text { Reference } \\
\text { variable } \\
2\end{array}$ & Other variable(s) & $\begin{array}{l}\text { Coefficient (healthcare costs per } \\
\text { patient) } \\
4\end{array}$ & $\begin{array}{l}\text { Coefficient (drug costs per } \\
\text { patient) } \\
5\end{array}$ \\
\hline $\begin{array}{l}\text { Follow-up completed vs. } \\
\text { dropout }\end{array}$ & Dropout & Follow-up completed & $(1.463)^{* *}$ & $(1.141)^{* *}$ \\
\hline Follow-up days & - & Follow-up days & 1161 & $1789 * *$ \\
\hline \multirow[t]{2}{*}{ Geographical area } & \multirow[t]{2}{*}{ Central region } & Northern region & 170.5 & 102.5 \\
\hline & & Southern region & $704.3^{* *}$ & $722.7 * * *$ \\
\hline Sex & Male & Female & $(393.0)$ & $(237.6)$ \\
\hline Age & - & Age & 11.77 & 15.58 \\
\hline \multirow[t]{7}{*}{ Comorbidity } & \multirow[t]{7}{*}{ None } & 3 & $1634 * *$ & $1228 * *$ \\
\hline & & 2 (diabetes + hypertension) & 889.3 & 392.3 \\
\hline & & 1 (dibetes) & 432.0 & 448.0 \\
\hline & & 2 (dyslipidaemia + hypertension) & 462.6 & 128.3 \\
\hline & & 1 (dyslipidaemia) & 203.9 & 234.1 \\
\hline & & 1 (hypertension) & 499.8 & 205.9 \\
\hline & & 2 (diabetes + dyslipidaemia) & 322.5 & 795.8 \\
\hline \multirow[t]{5}{*}{ CKD stage } & \multirow[t]{5}{*}{1} & 2 & 217.4 & 184.2 \\
\hline & & $3 a$ & 221.8 & 224.7 \\
\hline & & $3 b$ & $1095 * * *$ & $828.3 * * *$ \\
\hline & & 4 & $2501 * * *$ & $2027 * * *$ \\
\hline & & 5 & $3606 * * *$ & $2742 * * *$ \\
\hline \multirow[t]{5}{*}{ Proteinuria } & \multirow[t]{5}{*}{ Unknown } & Yes & (954.9) & $(1063)^{*}$ \\
\hline & & No & (725.6) & $(1026)^{*}$ \\
\hline & & Constant & 744.5 & 61.01 \\
\hline & & $N$ & 864 & 864 \\
\hline & & $R$-squared & 0.165 & 0.136 \\
\hline
\end{tabular}

$C K D$ chronic kidney disease

$* * * p<0.01, * * p<0.05, * p<0.01$

variables of CKD cost variations is CKD stage - the higher the CKD stage, the higher the unit cost per patient. Unit costs rocket when patients move from stage $3 a$ to stage $3 b$ and from stage $3 b$ to stage 4 . An increased effort should be made to maintain patients at levels $3 \mathrm{a}$ and $3 \mathrm{~b}$ in order to avoid cost escalations in the future.

Drugs represent the main component of CKD healthcare costs. Considering that (1) they avoid future costs and (2) drug unit costs also include pharmaceuticals for comorbidities (hypercholesterolemia, diabetes and dyslipidaemia), prevention of dialysis through an appropriate management of drug therapy seems a value-for-money investment.

Some variations in costs have been found across regions; however, differences across areas are not as huge as differences across CKD stages. Furthermore, geographical area was one of the most important explanatory variables. This aspect has not been further investigated, but it is likely that drug prescriptions should be better managed. In the southern region where healthcare unit costs are higher, the incidence of drugs on total healthcare expenditure is higher. Investment in drugs provides value for money, but drugs should be appropriately managed to avoid wasting resources.

In brief, this prospective observational study has provided important data to policy makers. Avoiding progression towards severe CKD and dialysis, together with a more appropriate drug prescription, would imply thousands of Euros saved, together with benefits to patients and society as a whole.

Data Availability Statement Data are not available due to the property rights of healthcare centres that have collected these data.

Author Contributions Claudio Jommi participated in writing the protocol, coordinated the pharmacoeconomic analysis of the study, 
and wrote the paper. Patrizio Armeni carried out the regression analysis and revised the paper. Margherita Battista and Paolo Di Procolo elaborated the data and revised the paper. Giussepe Conte, Claudio Ronco, Mario Cozzolino, Gabriella Concas, Giussepe Remuzzi participated in writing the protocol, collected data, provided clinical interpretation of the research findings, and revised the paper. Anna Maria Costanzo and Umberto di Luzio Paparatti wrote the protocol, supported data collection and analysis, and revised the paper.

\section{Compliance with Ethical Standards}

The results presented in this paper are part of a broader Italian prospective cohort study (Chronic Kidney Disease Management in Italy-IRIDE). AbbVie participated in the design and conduct of the study, interpretation of the data, and review and approval of the manuscript, and also provided financial support for the study. The authors would like to thank Giuliana Gualberti for her substantial support, and Arianna Iorio for supporting the research team in the first phase of the study.

Disclosures Umberto di Luzio Paparatti is an Abbvie employee and may own Abbvie stock/options. Anna Maria Costanzo was an Abbvie employee at the time the study was conducted and may own stock/ options. Claudio Jommi, Patrizio Armeni, Margherita Battista, Paolo Di Procolo, Giuseppe Conte, Claudio Ronco, Mario Cozzolino, Gabriella Concas and Giuseppe Remuzzi declare that thay have no conflicts of interest.

Open Access This article is distributed under the terms of the Creative Commons Attribution-NonCommercial 4.0 International License (http://creativecommons.org/licenses/by-nc/4.0/), which permits any noncommercial use, distribution, and reproduction in any medium, provided you give appropriate credit to the original author(s) and the source, provide a link to the Creative Commons license, and indicate if changes were made.

\section{References}

1. Center for Disease Control and Prevention (CDC). Chronic kidney disease initiative - protecting kidney health. Atlanta, GA: US
Department of Health and Human Services, Centers for Disease Control and Prevention; 2015. http://www.cdc.gov/diabetes/ projects/pdfs/ckd_summary.pdf. Accessed 31 Oct 2017.

2. Levey AS, Atkins R, Coresh J, et al. Chronic kidney disease as a global public health problem: approaches and initiatives. A position statement from Kidney Disease Improving Global Outcomes. Kidney Int. 2007;72:247-59.

3. Couser WG, Remuzzi G, Mendis S, Tonelli M. The contribution of chronic kidney disease to the global burden of major noncommunicable diseases. Kidney Int. 2011;80:1258-70.

4. Pontoriero G, Pozzoni P, Vecchio LD, et al. International Study of Health Care Organization and Financing for renal replacement therapy in Italy: an evolving reality. Int J Health Care Financ Econ. 2007;7:201-15.

5. Cicchetti A, Ruggeri M, Codella P, Ridolfi A. I costi sociosanitari dell'insufficienza renale cronica. Farmeconomia e Percorsi Terapeutici. 2011;12(1):21-8.

6. Trivedi H. Cost implications of caring for chronic kidney disease: are interventions cost-effective? Adv Chronic Kidney Dis. 2010;17:265-70.

7. Wyld M, Lee CMY, Chadban SJ, et al. Cost of CKD in Australia. Nephrology. 2014;S19:423.

8. Marx S, Petrilla A, Hengst N, Lee WC. Economic burden of advanced chronic kidney disease (CKD) (pre-dialysis) in a German cohort with type 2 diabetes and without diabetes. Nephrol Dial Transplant. 2014;29(Suppl 3):iii127-8.

9. Bellelli S, Turchetti G. Do socioeconomic inequalities impact the social cost of chronic kidney disease in Italy? Value Health. 2015;18(7):A509.

10. Roggeri DP, Roggeri A, Salomone M. Chronic Kidney disease: evolution of healthcare costs and resource consumption from predialysis to dialysis in Piedmont Region, Italy. Adv Nephrol 2014 (Article ID 680737).

11. Roggeri A, Roggeri DP, Zocchetti C, et al. Healthcare costs of the progression of chronic kidney disease and different dialysis techniques estimated through administrative database analysis. J Nephrol. 2017;30(2):263-9.

12. National Kidney Foundation. KDOQI clinical practice guideline for diabetes and CKD: 2012 update. Am J Kidney Dis. 2012;60(5):850-86.

13. Decreto Ministero della Salute 18 ottobre 2012. Tariffe delle prestazioni di ricovero ed ambulatoriali. 2012. 